\title{
¿AFECTAN LAS REDES SOCIALES A NUESTRA EMPATÍA? UN ESTUDIO CON JÓVENES UNIVERSITARIOS
}

\author{
DO SOCIAL NETWORKS AFFECT OUR EMPATHY? \\ A STUDY WITH YOUNG UNIVERSITY STUDENTS \\ EST- CE QUE LES RÉSEAUX SOCIAUX AFFECTENT NOTRE EMPATHIE? \\ UNE ÉTUDE AUPRÈS DE JEUNES ÉTUDIANTS UNIVERSITAIRES \\ Raquel Lozano-Blasco, Marta Mira-Aladrén y Mercedes Gil-Lamata \\ Universidad de Zaragoza
}

\section{Resumen:}

El presente trabajo estudia la afección de las redes sociales a la empatía cognitiva y afectiva. Concretamente se centra en analizar la población de jóvenes universitarios estudiantes de educación y psicología. Con este fin se lleva a cabo una investigación de corte cuantitativo mediante un diseño correlacional basado en el uso de cuestionarios psicométricos. Tras el exhaustivo análisis de datos se ha obtenido que existe un uso adictivo a las redes sociales al mismo tiempo que se dan dificultades en la empatía. También se observa que esta situación se ha visto agravada como consecuencia de la actual coyuntura de pandemia mundial. No obstante, se contempla que conforme aumenta la edad y se poseen mejores relaciones sociales, se dan menores dificultades en el componente cognitivo de la empatía. Por último, se obtiene que a pesar de la gran evidencia científica de anteriores estudios en los que se relaciona la adicción a redes sociales con la ansiedad, el presente estudio aboga por su no significancia. Esta circunstancia es aún más habitual en el sexo masculino, siendo el sexo femenino más sensible a padecer dicha situación.

Palabras clave: adicción; empatía; jóvenes; redes sociales; universitarios. 


\begin{abstract}
This paper studies the effect of social networks on cognitive and affective empathy. Specifically, it focuses on analyzing the population of young university students studying education and psychology. To this end, a quantitative research was carried out using a correlational design based on the use of psychometric questionnaires. After an exhaustive analysis of the data, it was found that there is an addictive use of social networks at the same time as there are difficulties in empathy. It is also observed that this situation has been aggravated as a consequence of the current global pandemic situation. However, it is observed that as age increases and social relationships improve, there are fewer difficulties in the cognitive component of empathy. Finally, it is found that despite the strong scientific evidence from previous studies linking social network addiction with anxiety, the present study argues for its non-significance. This circumstance is even more common in the male sex, with the female sex being more sensitive to this situation.
\end{abstract}

Keywords: addiction; empathy; youth; social networking sites; university students.

\title{
Résumé
}

Ce document étudie l'effet des réseaux sociaux sur l'empathie cognitive et affective. Plus précisément, elle se concentre sur l'analyse de la population des jeunes étudiants universitaires en éducation et en psychologie. À cette fin, une recherche quantitative est menée à travers un design corrélationnel basé sur l'utilisation de questionnaires psychométriques. Après l'analyse exhaustive des données, il a été obtenu qu'il existe une utilisation addictive des réseaux sociaux en même temps que des difficultés d'empathie. Il est également observé que cette situation s'est aggravée en raison de la situation actuelle de pandémie mondiale. Toutefois, on observe qu'à mesure que l'âge augmente et que les relations sociales s'améliorent, les difficultés liées à la composante cognitive de l'empathie diminuent. Enfin, on obtient que malgré la grande évidence scientifique des études précédentes dans lesquelles la dépendance aux réseaux sociaux est liée à l'anxiété, la présente étude plaide pour sa non-significativité. Cette situation est encore plus fréquente chez le sexe masculin, le sexe féminin étant plus sensible à cette situation.

Mots-clés: addiction; empathie; jeunes; réseaux sociaux; étudiants universitaires. 


\section{MARCO TEÓRICO}

El uso adictivo de las redes sociales se ha demostrado que afecta a determinadas cuestiones vinculadas a las habilidades interpersonales y de comunicación y, por ende, a su empatía y su capacidad para detectar las emociones de los demás (Carrier et al., 2015). Este hecho es especialmente relevante en futuros profesionales cuyas funciones están íntimamente relacionadas con esta competencia, siendo empleada como una de las herramientas básicas de trabajo, como puede ser enfermería, trabajo social, medicina, educación o psicología (Cusatis et al., 2019; Martínez-Otero, 2011; Extremera y Fernández, 2004). En lo referente a este aspecto, resulta trascendente averiguar cómo la adicción a Internet puede estar mediada tanto por la empatía como por la ansiedad, y las relaciones sociales medidas por el sociotipo.

En primer lugar, es necesario clarificar que la adicción a Internet es una de las patologías mentales que más se están extendiendo, atendiendo al meta-análisis de Cheng y Li (2014), la prevalencia crece conforme pasan los años. La adicción a Internet fue desarrollada por primera vez por Young (1998) exponiendo el creciente malestar de la población ante el uso de las nuevas tecnologías. Esta patología adolece de un criterio diagnóstico globalizado, pues ni la Organización mundial de la salud (OMS, 1992) ni la Asociación Americana de Psiquiatría (APA, 2013) la reconocen como tal.

La literatura académica apuesta por definirla desde la concepción clásica de Young (1998), es decir, la adicción conductual con la metáfora de hombre-máquina. Por otra parte, es necesario señalar la falta de cohesión en cuanto al rol de variables moderadoras. En cuanto al sexo, existe una elevada disparidad, por un lado, autores como Marino et al. (2018), Kuss et al. (2013) y Müller et al. (2014) exponen que no existe relación significativa, mientras que Černja et al. (2019) y Schimmenti et al. (2017) refieren justo lo opuesto. En cuanto a la edad, en rasgos generales la comunidad científica sostiene que esta es un elemento protector hacia la adicción (Li et al., 2010; Liu et al., 2017) si bien otros autores señalan su no significancia, mostrando una vi- 
sión no homogénea (Kuss y Griffiths, 2017).

La literatura académica expone la innegable comorbilidad que tiene la adicción a Internet con otras patologías como depresión, ansiedad, trastornos internalizantes y externalizantes, insomnio, traumas infantiles, etc. (Brailovskaia et al., 2019; Quílez-Robres et al., 2021; Lozano et al., 2020; Lu et al., 2018; Tas, 2019). De manera específica, las personas que padecen adicción a Internet y ansiedad exponen cómo las redes sociales retroalimentan la sintomatología ansiosa. Esto se debe a que refuerzan de manera constante la comparación social, y el estar permanentemente conectado a la espera de que suceda algo, más conocido como FOMO (Fear Of Missing Out, miedo a quedarse fuera); lo que afecta a su vida cotidiana (Andreassen et al., 2015; Alt y Boniel-Nissim, 2018; Wąsiński y Tomczyk, 2015). A este respecto hay que resaltar que no todas las investigaciones señalan esta relación, existiendo diferencias en la comunidad científica (Labrague, 2014). En consecuencia, la adicción a Internet y ansiedad se correlaciona de manera negativa con la satisfacción y calidad de vida, menguando el bienestar de la población (Cheng y Li, 2014). En lo relativo a la ansiedad, se debe clarificar que la relevancia sobre las variables sociodemográficas no es clara, si bien, la comunidad científica expone en su mayoría que las mujeres jóvenes están más afectadas (Andreassen et al., 2015; Dempsey et al., 2019; GarcíaDomingo et al., 2017; Lozano et al., 2020).

Las redes sociales incorporan de manera unívoca las habilidades interpersonales y de comunicación, es decir, la capacidad de la persona para relacionarse con su entorno (Carrier et al., 2015). Desde la perspectiva del cerebro social, el sociotipo, entendido como el entorno social y las interacciones sociales a las que los ser humanos están evolutivamente adaptados, resulta transcendente en actividades como son compartir, dar "me gusta", generar valor mediante "stories" o "ticktoks". A su vez, el sociotipo, del mismo modo que ocurre con el genotipo o el fenotipo a nivel biológico, afecta al desarrollo de nuestro cerebro y capacidades neurocognitivas (Marijuán et al., 2017). En este sentido, destaca el estudio de Gutiérrez-Puertas et al. (2020) y el de Carrier et al. (2015) en los que el uso inadecuado o excesivo de What- 
sapp y de videojuegos, respectivamente, se detecta como una de las causas de la disminución del aislamiento e incluso la empatía en estudiantes. Sin embargo, otros estudios manifiestan lo opuesto. La investigación realizada por Ayala (2012) explica como Facebook favorece la comunicación gracias a las opciones de "me gusta", "me encanta" y a la posibilidad de redactar comentarios. De este modo Facebook permite manifestar una respuesta empática que favorece y refuerzan las relaciones sociales (Ayala, 2012).

Aunque es manifiesta la relación vinculante entre adicción a internet, ansiedad y relaciones sociales (sociotipo), una de las cuestiones que más preocupan es si verdaderamente las nuevas tecnologías de la comunicación favorecen la disminución de la empatía (Ayala, 2012; Carrier et al., 2015; Gutiérrez-Puertas et al., 2020). ¿Cómo una actividad que a priori permite comunicarse y compartir podría llegar a afectar a un elemento tan humano como es la empatía? Tradicionalmente, la empatía ha sido estudiada a través de una perspectiva multidimensional. En ella se determinan dos variables: la empatía afectiva y la empatía cognitiva, las cuales son complementarias e imprescindibles para el desarrollo saludable psicosocial (Fillipeti et al., 2012; Shamay-Tsoory et al., 2009). No obstante, se dan dos supuestos contrarios, el primero es un sistema que defiende el contagio emocional a través de neuronas espejo con origen en la empatía afectiva (siendo un prerrequisito para la empatía cognitiva); y el segundo es un sistema exclusivo donde cada variable tiene circuitos neurales distintos, que a pesar de ser complementarios están disociados, actuando por separado en las estructuras neurales) (Fillipeti et al., 2012; Shamay-Tsoory et al., 2009).

Pese a esto, el sistema de exclusividad apoya su evidencia en pacientes con daños cerebrales y trastornos psicológicos como esquizofrenia, precisando que el déficit empático depende de las características del trastorno psicológico actual (FiIlipeti et al., 2012). Así pues, no hay un déficit común y genérico, de tal forma que en función de las estructuras afectadas se generará un cuadro distinto, aunque son las zonas de la corteza prefrontal las que mayor incidencia presentan (Decety y Mori- 
guchi, 2007; Fillipeti et al., 2012). En este sentido es importante tener en cuenta que la región prefrontal ventromedial es crítica en la empatía cognitiva y el giro frontal inferior en la empatía afectiva (Fillipeti et al., 2012).

Por otro lado, las actividades que implican una exposición significativa a actividades que involucran al sistema visual, como por ejemplo los videojuegos o las redes sociales, presentan patrones neuronales más maduros en el sistema visual (Paulus et al., 2019), si bien estos cambios pueden reducir el control cognitivo (Horowitz-Kraus y Hutton, 2017). Por otra parte, existen evidencias de cómo las actividades en pantalla se relacionan fuertemente con la inteligencia cristalizada, estando fuertemente influenciadas por la educación y la sociedad (Akshoomoff et al., 2013). Estos cambios son fruto de la novedad evolutiva y están guiados por la especialización (Sotiras et al., 2017).

A su vez, existen diferencias entre estudios en relación con la variable sexo. Por un lado, aquellos que afirman una relación negativa estadísticamente significativa entre la empatía en el mundo real con el aumento del tiempo tras una pantalla en el caso de las mujeres (Carrier et al., 2015), así como una mayor puntuación de las mujeres en fantasía, preocupación por los demás y sentirse mal ante situaciones empáticas con los demás (Ruiz Baca, 2013; Tobón et al., 2014; Pastor, 2004). En cambio, los estudios denotan mayores habilidades de comunicación por parte de los hombres (Gutiérrez-Puertas et al., 2020). Y, por otro lado, estudios donde se refleja que esta diferencia no es estadísticamente significativa. Por ejemplo, dentro del mismo estudio de Carrier et al. (2015), se concluyó que las puntuaciones de empatía cognitiva fueron más altas que las referentes a la empatía afectiva para ambos sexos, limitando así la diferencia entre ambos. Y el de Ruiz Baca (2013) encontró que no existían diferencias significativas entre sexos en las escalas de adopción de perspectivas y comprensión emocional.

Pese a estas diferencias en este contexto, conviene resaltar que existe un mayor consenso con respecto a la variable edad. Los estudios recabados concluyen que, tal y como apuntaban algunos autores clásicos, la edad sí que constituye una 
variable significativa en la empatía, especialmente la empatía cognitiva, incrementándose ésta a mayor edad (Arango et al., 2014; Retuerto, 2004). A su vez, destacan la influencia de la formación en el desarrollo de la empatía y la necesidad de desarrollarla en los currículos académicos, así como la relevancia de la socialización en la misma. A mayor socialización se daría una mayor empatía (Gutiérrez-Puertas et al., 2020; Cusatis et al., 2019; Arango et al., 2014; Ayala, 2012; Martínez-Otero, 2011; Extremera y Fernández, 2004).

La empatía es una variable clave en el proceso de socialización, si bien vale preguntarse si la nueva ecología que suponen las redes sociales, y el cambio conductual, así como cognitivo que implican afecta de alguna forma a esta variable.

El objetivo del estudio es determinar cómo afecta la adicción a las redes sociales a factores de empatía (cognitivos y afectivos), en los índices de ansiedad y en las relaciones sociales (sociotipo). La hipótesis de partida es que existe una interrelación compleja de las variables, de tal modo que la adicción a las redes sociales afecta directamente en los factores cognitivos y afectivos de empatía, así como en los índices de ansiedad y las relaciones sociales.

\section{METODOLOGÍA}

\section{Muestra}

La población de estudio se compone de un total de 100 jóvenes universitarios españoles con una edad media de 22,69 años, siendo el 50\% hombres y el 50\% mujeres. La muestra se obtuvo de la Universidad Internacional de Valencia $(\mathrm{N}=50,50 \%$ hombres, edad media=19,5, primer curso de Magisterio en Educación Infantil) y de la Universitat Oberta de Catalunya ( $N=50,50 \%$ hombres, edad media $=25,88$, tercer curso de Psicología). Esta muestra se recogió durante noviembre de 2020, siendo necesario resaltar este dato por encontrarse en un momento histórico marcado por la pandemia de Covid-19. Se trata de una muestra de conveniencia, se accedió a la misma mediante grupos cooperativos de la red social de Facebook de asignaturas concretas. 


\section{Diseño y procedimiento}

Se planteó una investigación de corte cuantitativo mediante un diseño correlacional basado en el uso de cuestionarios psicométricos. Los fenómenos y variables fueron analizados en su contexto natural, sin intervenir ni manipular previamente ninguno de ellos. Se elaboró un cuestionario ad hoc basado en instrumentos preexistentes y ya validados en la bibliografía científica, que se detallan más adelante. Dicho cuestionario se envió mediante la aplicación Formularios de Google a través de los grupos cooperativos de la red social de Facebook seleccionados en la definición de la muestra. En dichos cuestionarios se solicitaron los correos administrativos e institucionales, así como los consentimientos informados. El cuestionario fue totalmente anónimo. Tanto el procedimiento llevado a cabo como el posterior tratamiento de datos, se orientaron siguiendo los principios éticos establecidos en la Declaración de Helsinki de la Asociación Médica Mundial (WMA). De este modo, el cuestionario fue anónimo, voluntario, garantizando la confidencialidad establecida en la Declaración de Helsinki (World Medical Association, 2001). De la misma forma, se les expuso su derecho a revocar en cualquier momento su participación sin necesidad alguna de explicar la misma.

\section{Instrumentos}

Cuestionario de adicción a redes sociales (ARS) de Escurra y Salas (2014). La escala se compone de 24 ítems de tipo Likert con un intervalo de 5 puntos, desde O "nunca" hasta 4 "siempre". Dicho cuestionario "aporta evidencias de validez de constructo, bajo el modelo de la teoría clásica de los test a través del análisis factorial confirmatorio" (Escurra y Salas, 2014, p. 86). A este respecto, los correspondientes coeficientes alfa de Cronbach para cada uno de los tres factores, son indicadores de una fiabilidad alta (a F1=0.91; a F2=0.88; a F3=0.92). De igual modo, la consistencia interna de toda la escala es alta ( $a=0.94)$.

Inventario de Ansiedad de Beck (Test BAI) (Beck y Steer, 1988, adaptado al castellano por Sanz et al., 2011). Es un instrumento de autoinforme conformado por 21 ítems que fue inicialmente creado para analizar la gravedad de la sintomatología 
ansiosa. Se mide mediante una escala tipo Likert donde O significa "nada en absoluto" y 3 significa "gravemente, no podría soportarlo". Los valores de su consistencia interna y validez de constructo son excelentes. Concretamente, en la muestra que utilizan para la adaptación obtienen un índice alfa de Cronbach de 0.91 (Consejo General de Colegios Oficiales de Psicólogos, 2015).

Subescala Toma de Perspectiva (TP) de la adaptación al español del Interpersonal Reactivity Index (IRI) de Davis (1980). Se compone de 7 ítems con una valoración en escala tipo Likert de 1 a 5, siendo 1 No me describe bien y 5 Me describe muy bien. Este instrumento mide la capacidad de adoptar el punto de vista de otras personas. En cuanto a sus propiedades psicométricas éstas son buenas, teniendo un valor de alfa de Cronbach de la escala de 0.69 (Hogan, 1969).

Escala de Reactividad Emocional según el EQ Empathy Quotient (EQ) de Baron-Cohen y Wheelwright (2004), adaptación española de Redondo y Herrero-Fernández (2018), compuesta por 12 ítems, con una escala de valoración tipo Likert de 4 puntos (1=nunca me pasa a 4=siempre me pasa). Los valores alfa de Cronbach para todas las subescalas oscilaron entre .67 y .80. Esta variable estudia "la tendencia a tener una reacción emocional en respuesta a los estados mentales del otro, por ejemplo "ver a la gente Ilorar no me pone triste" (Redondo y Herrero-Fernández, 2018, p. 82).

Versión Española del "Cuestionario de Sociotipo" (SOCQ) (Marijuán et al., 2017). Se estructura en 16 ítems, con una escala Likert de 6 opciones de respuesta siendo, 0 "nunca" y 5 "siempre", salvo en los ítems 10 y 12 cuya valoración es inversa. Este instrumento estudia el concepto de sociotipo en diferentes roles: amistades, trabajo, conocidos y familia. En cuanto a sus propiedades psicométricas, presenta una validez y fiabilidad muy elevadas (Marijuán et al., 2017).

\section{Análisis estadísticos}

El análisis de datos se llevó a cabo mediante el programa JASP 0.14. Se aplicaron técnicas de análisis descriptivas de los resultados obtenidos en cada uno de los factores del ARS y en el Test BAI. Seguidamente, se estudió la relación entre la 
adicción a las redes y la ansiedad, y entre estas y las variables personales de sexo y edad. El análisis de regresión lineal múltiple permitió, finalmente, estudiar la influencia de unas variables sobre otras, respondiendo así a la finalidad última del estudio.

En primer lugar, se realizó un estudio descriptivo para determinar si la muestra seguía los parámetros de la curva normal, seguido de un estudio de medias y desviaciones típicas y un análisis de diferencias entre las modalidades de estudio de la muestra. Posteriormente se realizó un estudio de correlaciones de Pearson donde solo se tuvo en cuenta los resultados estadísticamente significativos, es decir aquellos en los que el valor $\mathrm{p}<0,05$, permitiendo aceptar la hipótesis alternativa. $A$ continuación, se realizó una regresión con pasos hacia delante para determinar y excluir las variables no significativas. Finalmente, se efectuó un análisis de mediación, para estudiar explicar el porqué y el cómo de la variable de adicción a redes sociales, siendo esta mediada por las variables emocionales de reactividad emocional y toma de perspectiva.

\section{RESULTADOS}

En primera instancia en la Tabla 1 se presentan las puntuaciones medias de las pruebas psicométricas realizadas encontrando que los resultados de la ansiedad (Media=16.71, DS=12.24) muestran una tasa normotípica, al igual que el sociotipo (Media=57,38, DS=10,33), mientras que la adicción a redes sociales (Media=52.64, DS=15.62) presenta una tasa elevada, que pone en riesgo a la población, sin llegar a ser esta patológica. Por otra parte, las puntuaciones medias de la empatía cognitiva o toma de perspectiva (Media=28,72, DS=8,33) y el componente emocional de la empatía o reactividad emocional (Media=42,97, DS=7,17) muestran tasas superiores a la media normotípica. Por otra parte, las pruebas estadísticas de Xi cuadrado mostraron que no había diferencias entre ambas universidades. En lo referente al sexo, las pruebas de T-Student mostraron ausencia de diferencias entre sexos salvo en la ansiedad ( $t=3.05, d f=99, p=0.003)$ encontrándose más afectado el sexo femenino. 
Tabla I.

Estadísticos descriptivos instrumentos psicométricos.

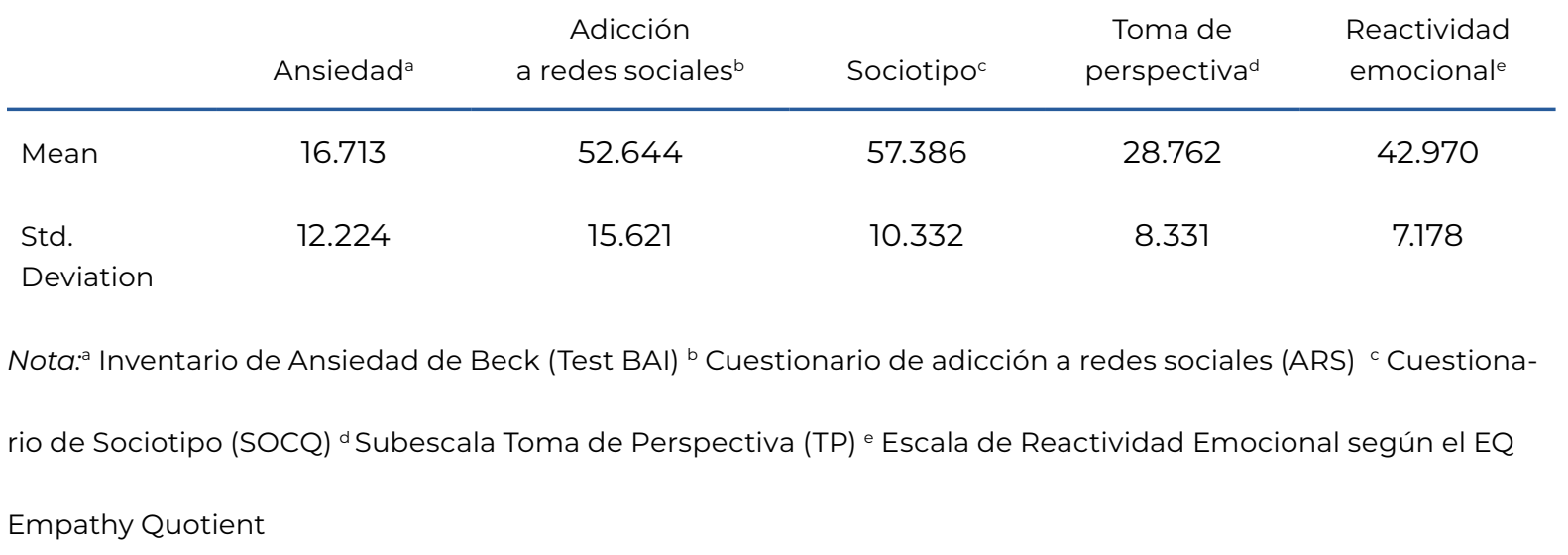

Se efectúa un análisis de correlaciones de Pearson para determinar la relación entre las diferentes variables, seleccionando únicamente aquellos datos con una significancia mayor a $\mathrm{p}<0,05$. Tal y como se puede ver en la Tabla 2, los resultados muestran una correlación negativa moderada entre adicción a redes sociales y toma de perspectiva (empatía cognitiva) $(r=-0,499, p<0,001)$, mientras que presenta una correlación positiva débil con la reactividad emocional $(r=0,289, p=0,003)$. Es decir, a medida que aumenta la adicción a las redes sociales disminuye la toma de perspectiva que permite comprender el estado mental de otras personas, mientras que aumenta la capacidad de reaccionar emocionalmente ante el estado emocional de otra persona. Por otra parte, la toma de perspectiva correlaciona positivamente con la variable edad $(r=0,362 ; p<0,001)$ y con el sociotipo $(r=0,233 ; p=0,019)$, es decir a mayor edad y mejores relaciones sociales se tendrá una mayor toma de perspectiva (empatía cognitiva). 
Tabla 2.

Estudio de correlaciones de Pearson.

\begin{tabular}{|c|c|c|c|c|c|c|c|}
\hline Variable & & Edad & Ansiedad $^{a}$ & $\begin{array}{l}\text { Adicción a re- } \\
\text { des sociales }^{b}\end{array}$ & Sociotipo $^{c}$ & $\begin{array}{c}\text { Toma de } \\
\text { perspectiva }^{d}\end{array}$ & $\begin{array}{l}\text { Reactividad } \\
\text { emocionale }\end{array}$ \\
\hline \multirow[t]{2}{*}{ 1.Edad } & Pearson's r & - & & & & & \\
\hline & $p$-value & - & & & & & \\
\hline \multirow[t]{2}{*}{ 2. Ansiedad } & Pearson's r & 0.125 & - & & & & \\
\hline & p-value & 0.211 & - & & & & \\
\hline \multirow{2}{*}{$\begin{array}{l}\text { 3.Adicción a } \\
\text { redes sociales }\end{array}$} & Pearson's r & 0,160 & 0.063 & - & & & \\
\hline & $p$-value & 0.109 & 0.532 & - & & & \\
\hline \multirow[t]{2}{*}{ 4.Sociotipo } & Pearson's r & 0.097 & 0.059 & -0.050 & - & & \\
\hline & $p$-value & 0.333 & 0.559 & 0.619 & - & & \\
\hline \multirow{2}{*}{$\begin{array}{l}\text { 5. Toma de } \\
\text { Perspectiva }\end{array}$} & Pearson's r & 0.362 & 0.145 & -0.499 & 0.233 & - & \\
\hline & $p$-value & $<.001$ & 0.147 & $<.001$ & 0.019 & - & \\
\hline \multirow{2}{*}{$\begin{array}{l}\text { 6. Reactividad } \\
\text { emociona }\end{array}$} & Pearson's r & 0.082 & 0.019 & 0.289 & -0.012 & -0.169 & - \\
\hline & p-value & 0.414 & 0.852 & v 0.003 & 0.905 & 0.092 & - \\
\hline
\end{tabular}

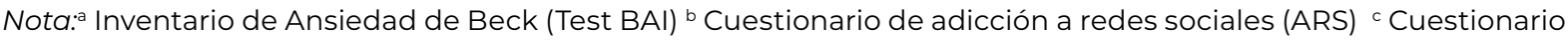
de Sociotipo (SOCQ) a Subescala Toma de Perspectiva (TP) e Escala de Reactividad Emocional según el EQ Empathy Quotient

Con la finalidad de determinar qué porcentaje de la varianza de la adicción a las redes sociales es explicada por las variables estudiadas anteriormente, se realizó una regresión con pasos hacia delante (ver Tabla 3 y 4), de tal forma que se eliminan aquellas variables que no eran significativas de manera progresiva. Aunque este método es más restrictivo aumenta la fiabilidad de los resultados estadísticos. De los modelos cuatro modelos, se opta por el modelo 4, el cual 29,2\% de la varianza $(R 2=0,292, p<0,001)$ de la adicción a redes sociales presentando una relación inversa con la toma de perspectiva (empatía cognitiva) ( $t=-5,36, p<0,001)$, y directa con la reactividad emocional $(t=2,42, p=0,016)$. 
Tabla 3.

Prueba ANOVA de la regresión múltiple con pasos hacia delante.

\begin{tabular}{|c|c|c|c|c|c|c|c|}
\hline Modelo & & $\begin{array}{c}\text { Suma } \\
\text { cuadrados }\end{array}$ & $\begin{array}{l}\text { de } \\
\text { df }\end{array}$ & $\begin{array}{c}\text { Media } \\
\text { cuadrado }^{b}\end{array}$ & $\mathbf{F}$ & $\mathbf{P}^{\mathbf{d}}$ & $\mathbf{R} 2^{\mathbf{e}}$ \\
\hline \multirow[t]{3}{*}{1.} & Regresión & 7610.762 & 5 & 1522.152 & 8.612 & $<.001$ & 0.312 \\
\hline & Residual & 16790.407 & 95 & 176.741 & & & \\
\hline & Total & 24401.168 & 100 & & & & \\
\hline \multirow[t]{3}{*}{2.} & Regresión & 7599.901 & 4 & 1899.975 & 10.856 & $<.001$ & 0.311 \\
\hline & Residual & 16801.267 & 96 & 175.013 & & & \\
\hline & Total & 24401.168 & 100 & & & & \\
\hline \multirow[t]{3}{*}{3.} & Regresión & 7515.235 & 3 & 2505.078 & 14.390 & $<.001$ & 0.308 \\
\hline & Residual & 16885.933 & 97 & 174.082 & & & \\
\hline & Total & 24401.168 & 100 & & & & \\
\hline \multirow[t]{3}{*}{4.} & Regresión & 7116.443 & 2 & 3558.222 & 20.470 & $<.001$ & 0.292 \\
\hline & Residual & 17284.725 & 98 & 176.375 & & & \\
\hline & Total & 24401.168 & 100 & & & & \\
\hline
\end{tabular}

Tabla 4.

Coeficientes de regresión múltiple con pasos hacia delante.

\begin{tabular}{|c|c|c|c|c|c|c|}
\hline Modelo & & No estandarizado & Error estándar & Estandarizado & $\mathrm{t}$ & $\mathrm{R} 2$ \\
\hline \multirow[t]{6}{*}{1.} & (Intercept) & 54.295 & 14.003 & & 3.877 & $<.001$ \\
\hline & Edad & -0.118 & 0.474 & -0.023 & -0.248 & 0.805 \\
\hline & Ansiedad & 0.165 & 0.110 & 0.129 & 1.499 & 0.137 \\
\hline & Sociotipo & 0.092 & 0.132 & 0.061 & 0.694 & 0.489 \\
\hline & Toma de Perspectiva & -0.916 & 0.180 & -0.488 & -5.077 & $<.001$ \\
\hline & Reactividad emocional & 0.450 & 0.190 & 0.207 & 2.361 & 0.020 \\
\hline
\end{tabular}


2.

(Intercept)

52.450

11.802

$4.444<.001$

Ansiedad

0.163

Sociotipo

Toma de Perspectiva

Reactividad emocional

3.

(Intercept)
Ansiedad
Toma de Perspectiva
Reactividad emocional
(Intercept)
Toma de Perspectiva
Reactividad emocional

$-0.092$

$-0.932$

0.442

56.759

0.165

$-0.905$

0.446

57.915

$-0.868$

0.458
0.110

0.132

0.167

0.187

10.018

0.109

0.163

0.187

10.055

0.162

0.188
0.128

1.492

0.139

0.061

$0.696 \quad 0.488$

$-0.947$

$-5.567<.001$

0.203

$2.362 \quad 0.020$

$5.666<.001$

$0.129 \quad 1.514 \quad 0.133$

$-0.483$

$-5.569<.001$

0.205

$2.389 \quad 0.019$

$5.760<.001$

$-0.463$

$-5.368<.001$

0.211

$2.442 \quad 0.016$

Atendiendo a la complejidad de la relación se efectuó un análisis de mediación que permitiera comprender cómo la variable adicción está mediada por las variables de la empatía, tanto cognitiva como emocional.

\section{Tabla 5.}

Efectos directos del análisis de mediación entre la adicción a redes sociales y las variables de empatía cognitiva (toma de perspectiva) y emocional (reactividad emocional).

\section{Efectos directos}

\begin{tabular}{|c|c|c|c|c|c|c|c|}
\hline & & \multirow[b]{2}{*}{ Estimación } & \multirow{2}{*}{$\begin{array}{l}\text { Error } \\
\text { Estándar }\end{array}$} & \multirow[b]{2}{*}{ Valor z } & \multirow[b]{2}{*}{$\mathbf{p}$} & \multicolumn{2}{|c|}{$\begin{array}{l}95 \% \text { Intervalo } \\
\text { de confianza }\end{array}$} \\
\hline & & & & & & Inferior & Superior \\
\hline Adicción a redes sociales & $\begin{array}{l}\rightarrow \text { Reactividad } \\
\text { emocional }\end{array}$ & 0.018 & 0.006 & 3.022 & 0.003 & 0.006 & 0.030 \\
\hline Adicción a redes sociales & $\begin{array}{l}\rightarrow \text { Toma de } \\
\text { Perspectiva }\end{array}$ & -0.032 & 0.005 & -6.056 & $<.001$ & -0.042 & -0.022 \\
\hline
\end{tabular}

Nota. Errores estándar del método Delta, intervalos de confianza de la teoría normal, estimador ML. 


\section{Tabla 6.}

Efectos indirectos del análisis de mediación entre la adicción a redes sociales, sociotipo o ansiedad y variables de empatía cognitiva (toma de perspectiva) y emocional (reactividad emocional)

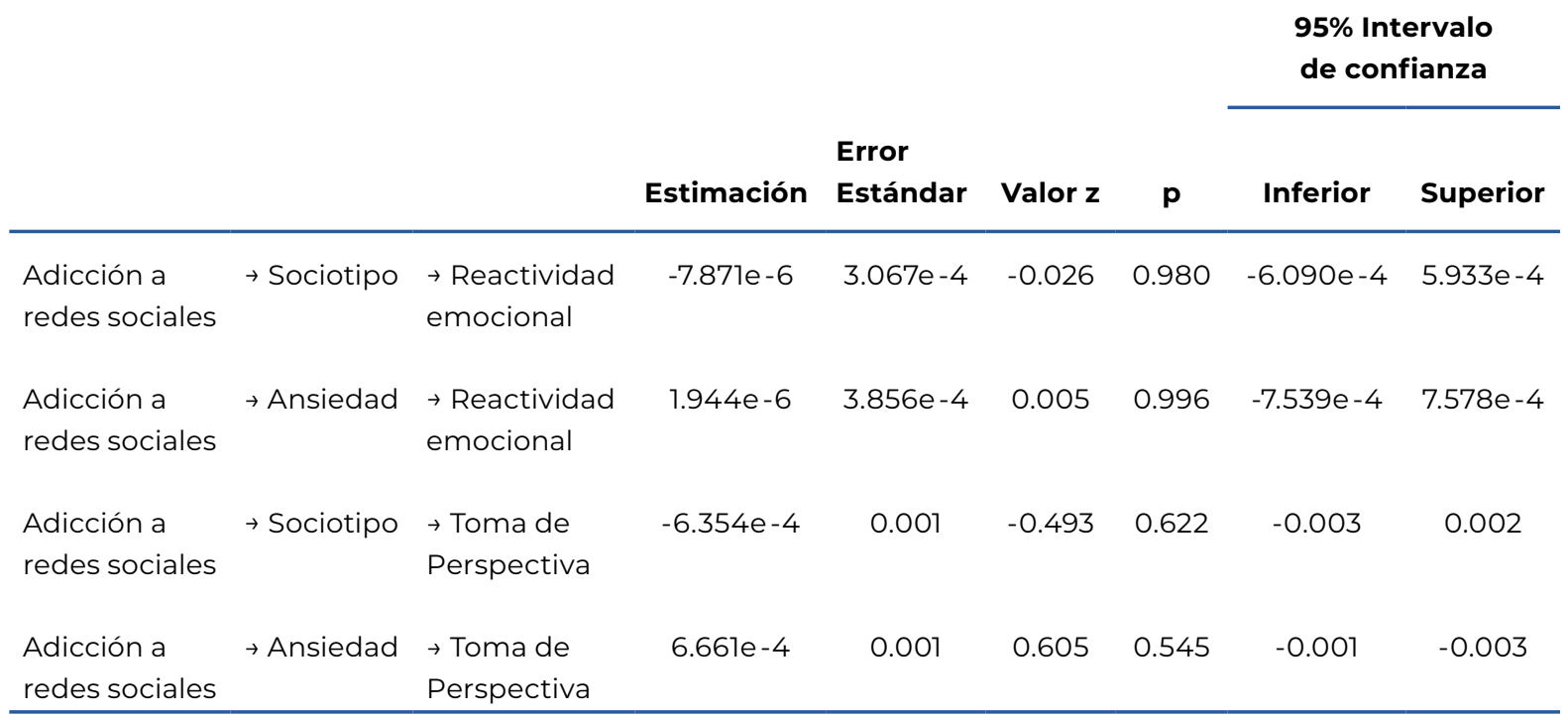

Nota. Errores estándar del método Delta, intervalos de confianza de la teoría normal, estimador ML.

\section{Tabla 7.}

Efectos totales del análisis de mediación entre la adicción a redes sociales y las variables de empatía cognitiva (toma de perspectiva) y emocional (reactividad emocional).

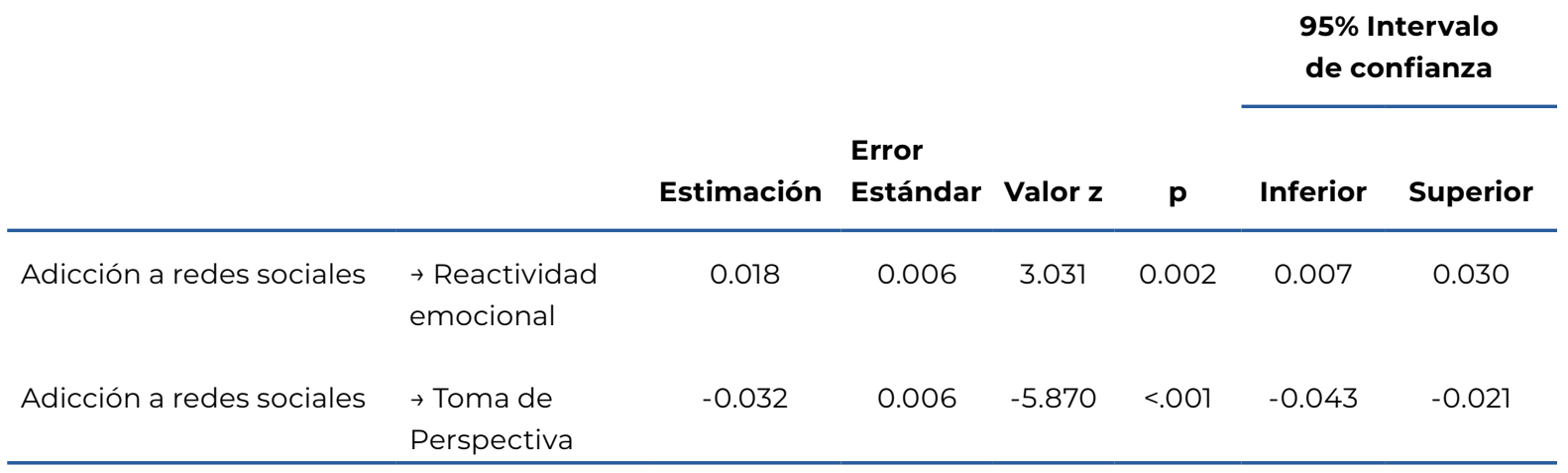

Nota. Errores estándar del método Delta, intervalos de confianza de la teoría normal, estimador ML. 
Tabla 8.

Covariables residuales del análisis de mediación

\begin{tabular}{|c|c|c|c|c|c|c|c|}
\hline & & \multirow[b]{2}{*}{ Estimación } & \multirow[b]{2}{*}{$\begin{array}{l}\text { Error } \\
\text { Estándar }\end{array}$} & \multirow[b]{2}{*}{ Valor $\mathbf{z}$} & \multirow[b]{2}{*}{$\mathbf{p}$} & \multicolumn{2}{|c|}{$\begin{array}{l}95 \% \text { Intervalo } \\
\text { de confianza }\end{array}$} \\
\hline & & & & & & Inferior & Superior \\
\hline Sociotipo & $\rightarrow$ Ansiedad & 0.061 & 0.098 & 0.624 & 0.533 & -0.131 & 0.254 \\
\hline Reactividad emocional & $\begin{array}{l}\rightarrow \text { Toma de } \\
\text { Perspectiva }\end{array}$ & -0.025 & 0.078 & -0.322 & 0.748 & -0.178 & 0.128 \\
\hline
\end{tabular}

Nota. Errores estándar del método Delta, intervalos de confianza de la teoría normal, estimador ML.

Los resultados de las Tablas 5, 6, 7 y 8 aparecen representados gráficamente en la Figura 1. Los coeficientes muestran que la ansiedad y el sociotipo no establecen relaciones mediadoras entre el proceso de adicción a redes sociales y las variables de reactividad emocional (empatía afectiva) y toma de perspectiva (empatía cognitiva). Es decir, la adicción a redes sociales no es fruto de la ausencia de relaciones sociales (sociotipo), ni de un cuadro ansioso. Éstos tampoco podrían considerarse causa o retroalimentación de cambios en la empatía humana. El propio proceso adictivo a las redes sociales altera directamente la capacidad empática humana, produciéndose un proceso complejo: favorece la reacción emocional fisiológica (reactividad emocional) pero frena el proceso de la comprensión de los procesos mentales de otras personas. Es decir, como fruto de este proceso existe un compromiso emocional con la situación de otras personas, pero, éste se combina con una dificultad para comprender el estado cognitivo de otras personas, esto supone que la población objeto de estudio no presenta desvinculación afectiva. 
Figura 1.

Trazado de la mediación entre variables de estudios

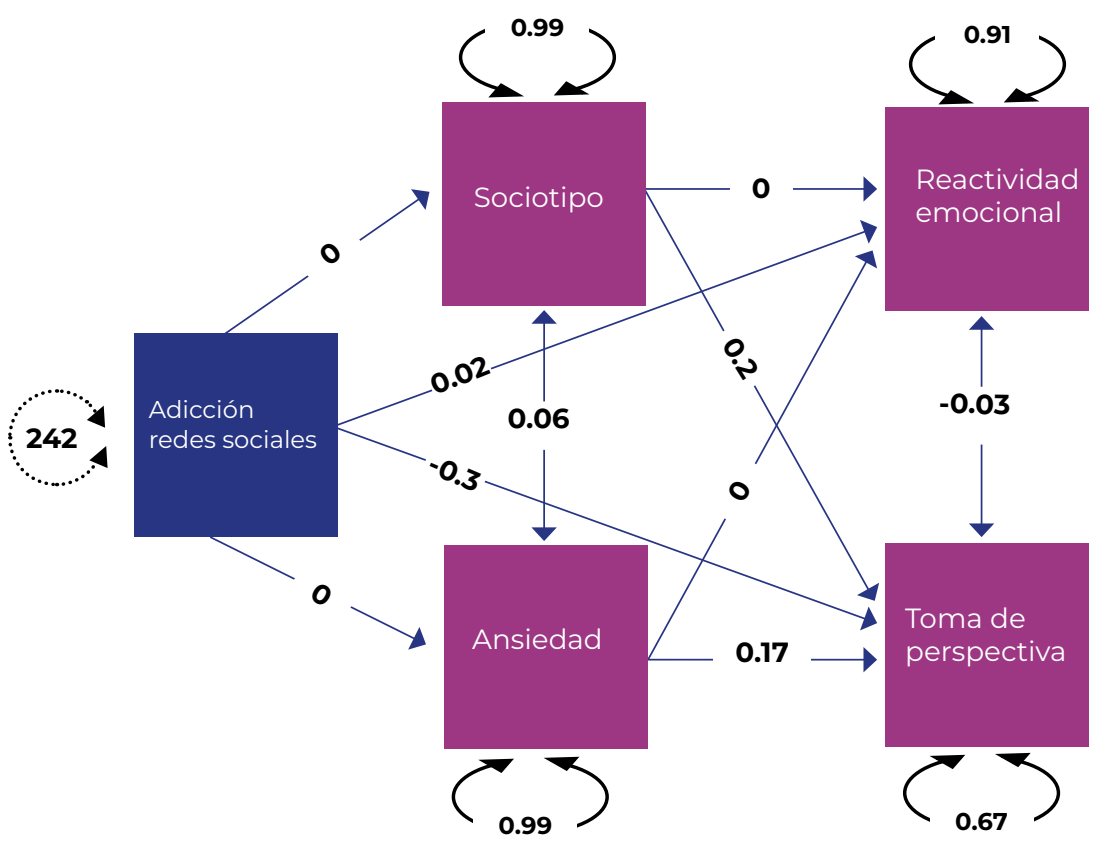

Fuente: Elaboración propia.

\section{DISCUSIÓN Y CONCLUSIONES}

La adicción a Internet y a las redes sociales es un gran reto al que se enfrenta la sociedad. De inicio, los resultados son coincidentes con investigaciones previas que señalan el aumento de la prevalencia de esta patología (Černja et al., 2019; Cheng y Li, 2014; Müller et al., 2014). Una de las mayores dificultades a las que se enfrenta el sistema sanitario y educativo es la comorbilidad elevada de la adicción junto a trastornos como la ansiedad, y a dificultades en habilidades sociales y empatía (Brailovskaia et al., 2019; Quílez-Robres et al., 2021; Lozano et al., 2020; Lu et al., 2018; Tas, 2019).

Sin embargo, a pesar de la fuerte evidencia científica que relaciona la adicción a redes sociales o Internet con la ansiedad (Lozano et al., 2020; Lu et al., 2018; Tas, 2019), nuestra investigación coincide con Labrague (2014) en su no significancia. Si bien, es necesario indicar la existencia de diferencias en materia de ansiedad en cuanto al sexo femenino siendo este más sensible a padecer estas situaciones, estos 
resultados son coherentes con investigaciones previas (Lozano et al., 2020). Por otra parte, se encontró que el sexo y la edad no resultaron variables determinantes en la adicción a las redes sociales estando en coherencia con estudios previos (Černja et al., 2019; Kuss y Griffiths, 2017; Schimmenti et al., 2017). Aunque los resultados de la presente investigación señalan la ausencia de comorbilidad, en materia de responsabilidad social se considera necesario apoyar e incentivar desde las universidades acciones y políticas públicas que refuercen la intervención en casos comórbidos.

En cuanto al sociotipo, los resultados muestran que la competencia a la hora de relacionarse no media en la adicción a Internet, es decir, la adicción a internet no presenta relación con dificultades sociales. De este modo, aunque las redes sociales sean un espacio de comunicación donde los usuarios comparten experiencias (Carrier et al., 2015; Gutiérrez-Puertas et al., 2020), las dificultades de relación con familia, amigos, compañeros de trabajo/estudio y conocidos de estos en la cotidianidad no se relacionan con comportamiento adictivo ni tampoco parecen generarlo. Estos resultados han de verse como un dato complementario a las investigaciones de Gutiérrez-Puertas et al. (2020) y el de Carrier et al. (2015) donde se expone cómo el uso adictivo de Whatsapp y de videojuegos favorece el aislamiento. En consecuencia, es necesario indagar más en esta temática para esclarecer el rol de las capacidades y habilidades sociales y comunicativas en las redes sociales. Igualmente, se plantea como estudio de prospectiva la necesidad de determinar si existen diferencias en habilidades sociales y comunicativas entre las relaciones presenciales versus virtuales.

En lo referente a la empatía, es necesario partir de la existencia de dos enfoques multidimensionales; el que aboga por un sistema básico de contagio mediante neuronas espejo o uno de exclusividad que expone que la empatía cognitiva y afectiva operan empleando diferentes conductos neurológicos (Fillipeti et al., 2012; Shamay-Tsoory et al., 2009). Si bien, la evidencia de este segundo modelo se da en población que padece dificultades de índole psicológica (Fillipeti et al., 2012; Shamay-Tsoory et al., 2009). De esta forma, los resultados de la presente investigación 
concuerdan con estudios previos en los que se señala la elevada comorbilidad entre el uso adictivo de redes sociales y dificultades en la empatía, confirmando la hipótesis de estudio y coincidiendo con estudios previos (Gutiérrez-Puertas et al., 2020; Carrier et al., 2015). En este sentido los resultados pueden vincularse con el auge del uso del smartphone y apps como Whatsapp, generando un descenso del contacto cara a cara (Gutiérrez-Puertas et al., 2020), debiendo considerarse que esta situación se agravó aún más con la situación derivada de la Covid-19 (Quílez-Robres et al., 2021; Gómez-Galán et al., 2020; Îñiguez-Berrozpe et al., 2020).

En cuanto al rol de las variables sociodemográficas de la empatía, los resultados del estudio coinciden con Carrier et al. (2015) y Ruiz Baca (2013) en la no significancia del sexo y con Retuerto (2004) en la similar capacidad cognitiva de ambos sexos para comprender la situación del otro/a y ponerse en su lugar. Además, encontramos en nuestros resultados que la toma de perspectiva (componente cognitivo de la empatía) se ve reforzado por la edad y por un sociotipo saludable, de manera que, a mayor edad, y mejores relaciones sociales existirán menos dificultades en el componente cognitivo de la empatía (Gutiérrez-Puertas et al., 2020; Cusatis et al., 2019; Arango et al., 2014; Ayala, 2012; Martínez-Otero, 2011; Extremera y Fernández, 2004; Retuerto, 2004). Como estudios prospectivos, se plantea la necesidad de realizar estudios semejantes con grupos control en una muestra poblacional normotípica y otra diagnosticada de adicción a Internet o redes sociales. También se plantea la necesidad de realizar estudios de revisión acerca de las terapias más afectivas en la mejora de la empatía para poder seleccionar y proponer un modelo interventivo ajustado a las características de una población con adicción a las redes sociales. Por último, se considera que sería de gran interés la realización de un estudio comparativo entre los estudiantes de diferentes cursos de una misma titulación.

El presente estudio tiene como principal limitación la causada por la excepcionalidad de la situación pandémica; teniendo en cuenta que las investigaciones de los últimos meses señalan que la empatía ha podido verse modificada a razón de la pandemia (Quílez-Robres et al., 2027; Lozano et al., 2020). Esta situación ha podido 
afectar a los procesos cognitivos y afectivos de la empatía, no sólo por la info-toxicación experimentada durante el confinamiento de la población, sino por el propio estrés e impotencia surgidos en esta etapa histórica.

\section{REFERENCIAS}

Akshoomoff, N., Beaumont, J. L., Bauer, P. J., Dikmen, S. S., Gershon, R. C., Mungas, D., Slotkin, J., Tulsky, D., Weintraub, S., Zelazo, P. D. y Heaton, R. K. (2013). VIII. NIH Toolbox Cognition Battery (CB): composite scores of crystallized, fluid, and overall cognition. Monographs of the Society for Research in Child Development, 78(4), 119-132. https://doi.org/10.1007/s40429-015-0056-9

Alt, D. y Boniel-Nissim, M. (2018). Links between Adolescents' Deep and Surface Learning Approaches, Problematic Internet Use, and Fear of Missing Out (FoMO). Internet Interv, 13, 30-39. https://doi.org/10.1016/j.invent.2018.05.002 American Psychiatric Association (APA) (2013). Diagnostic and statistical manual of mental disorders (5th ed.). Arlington.

Andreassen, C. S. (2015). Online social network site addiction: A comprehensive review. Curr. Addict. Rep., 2, 175-184. https://doi.org/10.1007/s40429-015-00569

Arango, O. E., Clavijo, S. J., Puerta, I. C. y Sánchez, J. W. (2014). Formación académica, valores, empatía y comportamientos socialmente responsables en estudiantes universitarios. Revista de la Educación Superior, XLIII(1) (169), 89-105.

Ayala, M. H. (2012). Expresión personal y empatía en las redes sociales: los estudiantes universitarios y el uso de Facebook. Cuadernos de H ideas, 6(6). http:// sedici.unlp.edu.ar/handle/10915/33167

Beck, A. T. y Steer, R. A. (1988). Manual. BAI. Inventario de Ansiedad de Beck. Adaptación española de Sanz, J., Vallar, F., De la Guía, E. y Hernández, A. (2011). Pearson Educación.

Brailovskaia, J., Margraf, J. y Köllner, V. (2019). Addicted to Facebook? Relationship between Facebook Addiction Disorder, duration of Facebook use and nar- 
cissism in an inpatient sample. Psychiatry Research, 273, 52-57. https://doi. org/10.1016/j.psychres.2019.01.016

Carrier, L. M., Spradlin, A., Bunce, J. P. y Rosen, L. D. (2015). Virtual empathy: Positive and negative impacts of going online upon empathy in young adults. Computers in Human Behavior, 52, 39-48. https://doi.org/10.1016/j. chb.2015.05.026

Černja, I., Vejmelka, L. y Rajter, M. (2019). Internet addiction test: Croatian preliminary study. BMC Psychiatry, 19(1), 1-11. https://doi.org/10.1186/s12888-0192366-2

Cheng, C. y Li, A. Y. (2014). Internet Addiction Prevalence and Quality of (Real) Life: A Meta-Analysis of 31 Nations Across Seven World Regions. Cyberpsychology, Behavior, and Social Networking, 17(12), 755-760. https://doi.org/10.1089/cyber.2014.0317

Consejo General de Colegios Oficiales de Psicólogos (2015). Código Deontológico del Consejo General de Psicología. https://www.cop.es/index.php?page=CodigoDeontologico

Cusatis, R., Holt, J. M., Williams, J., Nukuna, S., Asan, O., Flynn, K. E., ... y Crotty, B. H. (2019). The impact of patient-generated contextual data on communication in clinical practice: A qualitative assessment of patient and clinician perspectives. Patient Education and Counseling, 103(4), 734-740. https://doi. org/10.1016/j.pec.2019.10.020

Davis, M. H. (1980). A multidimensional approach to individual differences in empathy. JSAS Catalog of Selected Documents in Psychology, 10, 85.

Decety, J. y Moriguchi, Y. (2007). The empathic brain and its dysfunction in psychiatric populations: Implications for intervention across different clinical conditions. BioPsychoSocial medicine, 1(1), 1-21. https://doi.org/10.1186/17510759-1-22

Dempsey, A., O’Brien, K. D., Tiamiyu, M. F. y Elhai, J. D. (2019). Fear of missing out (FoMO) and rumination mediate relations between social anxiety and pro- 
blematic Facebook use. Addict. Behav. Rep., 9, 1-17. https://doi.org/10.1016/j. abrep.2018.100150

Escurra, M. y Salas, E. (2014). Construcción y validación del cuestionario de adicción a redes sociales (ARS). Liberabit, 20(1), 73-91.

Extremera, N. y Fernández, P. (2004). Inteligencia emocional, calidad de las relaciones interpersonales y empatía en estudiantes universitarios. Clínica y Salud, 15(2), 117-137.

Filippetti, V. A., López, M. B. y Richaud, M. C. (2012). Aproximación neuropsicológica al constructo de empatía: aspectos cognitivos y neuroanatómicos. Cuadernos de Neuropsicología/Panamerican Journal of Neuropsychology, 6(1), 63-83.

García-Domingo, M., Aranda, M. y Fuentes, V. M. (2017). Facebook Use in University Students: exposure and reinforcement search. Procedia - Soc. and Behav. Sci., 237, 249-254. https://doi.org/10.1016/j.sbspro.2017.02.071

Gómez-Galán, J., Martínez-López, J. Á., Lázaro-Pérez, C. y Sarasola, J. L. (2020). Social Networks Consumption and Addiction in College Students during the COVID-19 Pandemic: Educational Approach to Responsible Use. Sustainability, 12(18), 7737. https://doi.org/10.3390/su12187737

Gutiérrez-Puertas, L., Márquez-Hernández, V. V., Gutiérrez-Puertas, V., Granados-Gámez, G. y Aguilera-Manrique, G. (2020). Interpersonal communication, empathy, and stress perceived by nursing students who use social networks. Journal of Advanced Nursing, 76(10), 2610-2617. https://doi.org/10.1111/ jan.14494

Hogan, R. (1969). Development of an empathy scale. Journal of Consulting and Clinical Psychology, 33(3), 307-316. https://doi.org/10.1037/h0027580

Horowitz-Kraus, T. y Hutton, J. S. (2017). Brain connectivity in children is increased by the time they spend reading books and decreased by the length of exposure to screenbased media. Acta Paediatrica, 107, 685-693. https://doi. org/10.1111/apa.14176 
Íñiguez-Berrozpe, T., Cano-Escoriaza, J., Cortés-Pascual, A. y Elboj-Saso, C. (2020). Structural model of concurrence among relational bullying and cyberbullying: Victims, aggressors and bystanders. Revista Española de Investigaciones Sociológicas, 171, 63-84. https://doi.org/10.5477/cis/reis.171.63

Kuss, D. J. y Griffiths, M. D. (2017). Social networking sites and addiction: Ten lessons learned. International journal of environmental research and public health, 14(3), 311. https://doi.org/10.3390/ijerph14030311

Kuss D. J, Griffiths M. D. y Binder J. (2013). Internet addiction in students: prevalence and risk factors. Computers in Human Behavior, 29(3), 959-966. https:// doi.org/10.1016/j.chb.2012.12.024

Labrague, L. (2014). Facebook use and adolescents' emotional states of depression, anxiety, and stress. Health Science Journals, 8, 80-89. http://hdl.handle. net/11400/1481

Li, D., Zhang, W., Li, X., Zhen, S. y Wang, Y. (2010). Stressful life events and problematic Internet use by adolescent females and males: A mediated moderation model. Computers in Human Behavior, 26(5), 1199-1207. https://doi. org/10.1016/j.chb.2010.03.031

Liu, F., Zhang, J., Zhou, N., Li, X. y Fang, X., (2017). The relationship between family functions and college students' internet addiction: the mediating effect of coping styles. Chinese Journal of Special Education, 24(2), 90-96. https:// doi.org/10.1016/j.addbeh.2016.08.009

Lozano, R., Latorre, C. y Quílez-Robres, A. (2020). Social network addiction and its impact on anxiety level among university students. Sustainability, 12(13), 5397. https://doi.org/10.3390/su12135397

Lu, L., Liang, Z., Wu, L., Chen, Y., Song, Y., Dhanabalan, S. C., ... y Zhang, H. (2018). Few-layer bismuthene: sonochemical exfoliation, nonlinear optics and applications for ultrafast photonics with enhanced stability. Laser \& Photonics Reviews, 12(1), 1700221. https://doi.org/10.1002/Ipor.201700221

Marijuán, P. C., Montero-Marín, J., Navarro, J., García-Campayo, J. y Del Moral, 
R. (2017). The "sociotype" construct: Gauging the structure and dynamics of human sociality. PloS one, 12(12). https://doi.org/10.1371/journal. pone.0189568

Marino, C., Gini, G., Vieno, A. y Spada, M. M. (2018). A comprehensive meta-analysis on Problematic Facebook Use. Computers in Human Behavior, 83, 262-277. https://doi.org/10.1016/j.chb.2018.02.009

Martínez-Otero Pérez, V. (2011). La empatía en la educación: estudio de una muestra de alumnos universitarios. Revista Electrónica de Psicología Iztacala, 14(4), 174-190.

Müller, K. W., Beutel, M. E. y Wölfling, K. (2014). A contribution to the clinical characterization of Internet addiction in a sample of treatment seekers: validity of assessment, severity of psychopathology and type of co-morbidity. Comprehensive Psychiatry, 55, 770-777. https://doi.org/10.1016/j.comppsych.2014.01.010

Organización Mundial de la Salud (OMS) (1992). CIE-10. Trastornos Mentales y del Comportamiento. Décima Revisión de la Clasificación Internacional de las Enfermedades. Descripciones Clínicas y pautas para el diagnóstico. Organización Mundial de la Salud.

Pastor, Á. R. (2004). Diferencias en empatía en función de las variables género y edad. Apuntes de psicología, 22(3), 323-339.

Paulus, M. P., Squeglia, L. M., Bagot, K., Jacobus, J., Kuplicki, R., Breslin, F. J. y Tapert, S. F. (2019). Screen media activity and brain structure in youth: evidence for diverse structural correlation networks from the ABCD study. Neuroimage, 185, 140-153. https://doi.org/10.1016/j.neuroimage.2018.10.040

Quílez-Robres, A., Lozano-Blasco, R., Îniguez-Berrozpe, T. y Cortés-Pascual, A. (2021). Social, Family, and Educational Impacts on Anxiety and Cognitive Empathy Derived From the COVID-19: Study on Families With Children. Frontiers in Psychology, 12. http://dx.doi.org/10.3389/fpsyg.2021.562800

Redondo, I. y Herrero-Fernández, D. (2018). Adaptación del Empathy Quotient (EQ) 
en una muestra española. Terapia Psicológica, 36(2), 81-89.

Retuerto, A. (2004). Diferencias en empatía en función de las variables género y edad. Apuntes de psicología, 22(3), 323-339. https://hdl.handle. net/11441/84917

Ruiz Baca, P. (2013). Propiedades psicométricas del test de empatía cognitiva y afectiva en estudiantes no universitarios. Cátedra Villarreal, 7(1), 99-116.

Schimmenti, A., Passanisi, A., Caretti, V., La Marca, L., Granieri, A., lacolino, C., Gervasi, A. M., Maganuco, N. R. y Billieux, J. (2017). Traumatic experiences, alexithymia, and Internet addiction symptoms among late adolescents: A moderated mediation analysis. Addictive Behaviors, 64, 314-320. https:// doi.org/10.1016/j.addbeh.2015.11.002

Shamay-Tsoory, S. G., Aharon-Peretz, J. y Perry, D. (2009). Two systems for empathy: A double dissociation between emotional and cognitive empathy in inferior frontal gyrus versus ventromedial prefrontal lesions. Brain, 132, 617-627. https://doi.org/10.1093/brain/awn279

Sotiras, A., Toledo, J. B., Gur, R. E., Gur, R. C., Satterthwaite, T. D. y Davatzikos, C. (2017). Patterns of coordinated cortical remodeling during adolescence and their associations with functional specialization and evolutionary expansion. Proceedings of the National Academy of Sciences, 174, 3527-3532. https://doi.org/10.1073/pnas.1620928114

Tas, I. (2019). Association between Depression, Anxiety, Stress, Social Support, Resilience and Internet Addiction: A Structural Equation Modelling. MaIaysian Online Journal of Educational Technology, 7(3), 1-10. http://dx.doi. org/10.17220/mojet.2019.03.001

Tobón, O. E. A., Zapata, S. J. C., Lopera, I. C. P. y Duque, J. W. S. (2014). Formación académica, valores, empatía y comportamientos socialmente responsables en estudiantes universitarios. Revista de la educación superior, 43(169), 89105. https://doi.org/10.1016/j.resu.2015.01.003

Wąsiński, A. y Tomczyk, L. (2015). Factors reducing the risk of internet addiction in 
young people in their home environment. Child and Youth Serv. Rev., 57, 68-74. https://doi.org/10.1016/j.childyouth.2015.07.022

World Medical Association. (2001). World Medical Association Declaration of Helsinki. Ethical principles for medical research involving human subjects. Bulletin of the World Health Organization, 79(4), 373.

Young, K. S. (1998). Caught in the net: How to recognize the signs of internet addiction and a winning strategy for recovery. John Wiley \& Sons.

Fecha de recepción 15 de abril de 2021

\section{Fecha de aceptación 21 de septiembre de 2021}

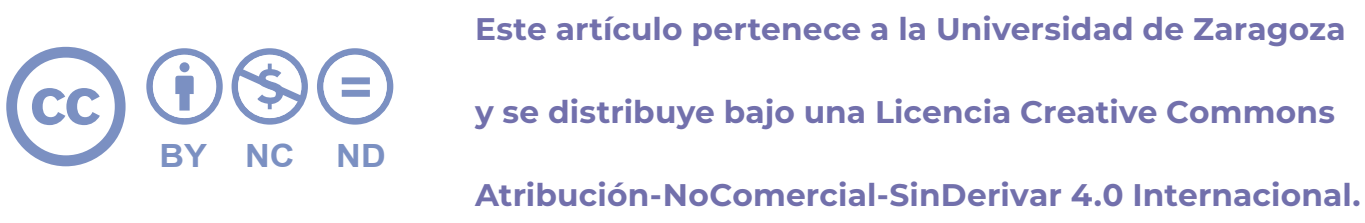

Eres libre de compartir copiar y redistribuir el material en cualquier medio o formato -

Bajo las condiciones siguientes:

- Reconocimiento de la autoría, ya incluida en esta diapositiva.

- NoComercial - no se puede utilizar el material para una finalidad comercial.

- SinObraDerivada - Sin remezclar, transformar o crear a partir del material. 